\title{
Instabilities of the harmonic oscillator with fluctuating damping
}

\author{
Vicenç Méndez, ${ }^{1}$ Werner Horsthemke, ${ }^{2}$ Pau Mestres, ${ }^{1}$ and Daniel Campos ${ }^{1}$ \\ ${ }^{1}$ Grup de Física Estadística, Departament de Física, Universitat Autònoma de Barcelona, 08193 Barcelona, Spain \\ ${ }^{2}$ Department of Chemistry, Southern Methodist University, Dallas, Texas 75275-0314, USA
}

(Received 15 July 2011; published 25 October 2011)

\begin{abstract}
We investigate the instabilities of a linear damped oscillator due to fluctuations of the damping parameter. The fluctuations are driven either by Gaussian white noise or Poisson white noise (white shot noise). We consider three notions of stability. The first two are the well-known notions of stability in the mean and stability in the mean square. We introduce the concept of thermodynamic stability, corresponding to a nonpositive rate of energy dissipation at all times. We derive analytical results for the various instability thresholds, confirm the validity of our approach for white shot noise by numerical simulations, and obtain the unexpected result that mean-square and thermodynamic stability coincide for the two types of white noise.
\end{abstract}

DOI: 10.1103/PhysRevE.84.041137

PACS number(s): 05.40.Ca, 05.10.Gg, 02.50.Ey

\section{INTRODUCTION}

The classical harmonic oscillator is a model system that has a history of more than a hundred years in the scientific literature and that is discussed in every first-year course in physics. Its has achieved the status of a paradigm because it describes the behavior of a wide variety of systems in physics, chemistry, biology, and other fields. To quote Gitterman [1], "In fact, it has been applied everywhere, from quarks to cosmology." Examples include mechanical systems such as pendula with small angles of displacement and mass-spring systems, acoustical systems and electric systems such as RLC circuits, as well as man-made devices such as clocks and radio circuits. The prominence and importance of this model stem from the fact that the dynamics of any system in a potential near a local minimum can be approximated by simple harmonic motion. The harmonic oscillator still holds surprises and remains the object of current research despite its long history.

If friction or energy dissipation has to be taken into account, one arrives at the damped harmonic oscillator where the forces are linearly proportional to the displacement from the local minimum and to the velocity:

$$
m \frac{d^{2} x}{d t^{2}}+f \frac{d x}{d t}+m \omega^{2} x=0 .
$$

The study of the effects of random fluctuations, or noise, on simple harmonic motion has also a long history (see, e.g., [1]). Noise can enter Eq. (1.1) in four ways. The simplest, and most thoroughly studied, case corresponds to Brownian motion of a harmonically bound particle [2,3]:

$$
m \frac{d^{2} x}{d t^{2}}+f \frac{d x}{d t}+m \omega^{2} x=\zeta(t)
$$

The noise $\zeta(t)$ enters Eq. (1.1) in an additive manner and represents internal thermal noise. Fluctuations in the potential energy give rise to a random frequency [4-7]:

$$
m \frac{d^{2} x}{d t^{2}}+f \frac{d x}{d t}+m \omega^{2}[1+\zeta(t)] x=0 .
$$

In this case, the noise affects the motion in a multiplicative way. The interplay of the fluctuations in the frequency with an external periodic driving force can give rise to stochastic resonance [8,9]. Fluctuations in the friction coefficient $f$ and the mass $m$ also lead to stochastic differential equations with multiplicative noise. The latter case has recently been studied to describe Brownian motion with adhesion [10,11].

If we adopt a mechanical picture, then fluctuations in the friction coefficient around a mean value arise due to random variations in the viscosity of the medium surrounding the particle. An example is a ferroelectric fluid whose viscosity is controlled by external stochastic magnetic fields as discussed in $[12,13]$, where the diffusion properties of Brownian particles with a fluctuating friction force were investigated. However, the stochastic differential equation describes a variety of systems, as discussed in $[1,9,14]$. It was applied for example to critical binary fluids [15], to the coupling between water waves and a turbulent wind field [16], and to motions of floating, moored offshore structures [17].

We study the effects of fluctuations in the damping coefficient on the stability of the oscillator. The mean position of the oscillator increases without bound in time if the noise intensity is high enough. Gitterman obtained this result for Gaussian white noise and colored noise, namely, dichotomous noise, by analyzing the evolution of the mean position [14]. He considered a somewhat different system in [18]; namely, the oscillator is also subjected to an additive random force. This random force is Gaussian, white, and not correlated with the multiplicative noise. For this system, he determined the stationary second moments if the damping coefficient displays Gaussian white noise or dichotomous noise. For the case of Gaussian white noise, the stationary second moments diverge at a certain noise intensity that is lower than the noise intensity threshold value for divergence of the mean position. No explicit expression for the instability threshold of the second moments was derived for the case of dichotomous noise.

These results illustrate that even if the mean position relaxes to zero, the oscillator can still be unstable, namely, if the mean-square position increases with time. It is necessary to distinguish between stability in the mean and stability in the mean square. We introduce a third notion of stability, namely, thermodynamic stability. The oscillator is thermodynamically stable if its rate of energy dissipation is nonpositive. We focus exclusively on the effects of multiplicative noise and consider a harmonic oscillator that is subject only to random damping; 
the oscillator does not experience an additive random force. We adopt a mechanical picture and formulate our approach within the framework of canonical-dissipative systems [19-21]. We derive exact analytic conditions for stability in the mean, in the mean square, and for thermodynamic stability for Gaussian white noise and white shot noise (Poisson white noise). Poisson white noise has the advantageous feature that it has a lower bound in contrast to Gaussian white noise. We can thus assess the effect of negative fluctuations, which change the nature of the friction term, on the stability results. We establish the surprising result that thermodynamic stability and stability in the mean square coincide for both types of white noise.

\section{CLASSICAL MECHANICS AND DISSIPATIVE SYSTEMS}

The equations of motion of a mechanical system with the Hamiltonian $H(x, p)$ are

$$
\begin{gathered}
\frac{d x}{d t}=\frac{\partial H}{\partial p}, \\
\frac{d p}{d t}=-\frac{\partial H}{\partial x} .
\end{gathered}
$$

As is well known, Hamiltonian systems are conservative systems that do not dissipate energy. From the same Hamiltonian we can construct, however, a system that includes dissipation by replacing Eq. (2.2) with

$$
\frac{d p}{d t}=-\frac{\partial H}{\partial x}-f(H) \frac{\partial H}{\partial p} .
$$

This is known as a canonical-dissipative system [19-21], and $f(H)$ denotes the dissipation function. The friction force $F$ of the canonical-dissipative system is given by

$$
F=-f(H) \frac{\partial H}{\partial p}=-f(H) \frac{d x}{d t} .
$$

A canonical-dissipative system does indeed not conserve energy:

$$
\begin{aligned}
\frac{d H}{d t} & =\frac{\partial H}{\partial x} \frac{d x}{d t}+\frac{\partial H}{\partial p} \frac{d p}{d t} \\
& =\frac{\partial H}{\partial x} \frac{\partial H}{\partial p}+\frac{\partial H}{\partial p}\left[-\frac{\partial H}{\partial x}-f(H) \frac{\partial H}{\partial p}\right] \\
& =-f(H)\left(\frac{d x}{d t}\right)^{2} \equiv \frac{d E}{d t},
\end{aligned}
$$

where we have made use of Eqs. (2.1) and (2.3). Equation (2.5) provides the rate of energy dissipation $d E / d t$, by a particle moving according to the equations of motion (2.1) and (2.3).

From Eqs. (2.1) and (2.3) we derive the equations of motion for a particle of mass $m$ moving in a parabolic potential by considering the Hamiltonian $H=p^{2} / 2 m+m \omega^{2} x^{2} / 2$ :

$$
\begin{gathered}
\frac{d x}{d t}=\frac{p}{m}, \\
\frac{d p}{d t}=-m \omega^{2} x-f(H) \frac{p}{m} .
\end{gathered}
$$

We combine these equations to obtain

$$
\frac{d^{2} x}{d t^{2}}+\frac{1}{m} f(H) \frac{d x}{d t}+\omega^{2} x=0 .
$$

If the dissipation function is a constant, $f(H)=m b$, Eq. (2.8) represents the classical linear damped harmonic oscillator, where $b$ is the damping coefficient. The linear damped harmonic oscillator dissipates energy with the rate

$$
\frac{d E}{d t}=-m b\left(\frac{d x}{d t}\right)^{2} .
$$

We use Eqs. (2.5) and (2.8) to derive an alternative expression for the rate of energy dissipation, which will prove helpful later on:

$$
\begin{aligned}
\frac{d E}{d t} & =m \frac{d x}{d t} \frac{d^{2} x}{d t^{2}}+m \omega^{2} x \frac{d x}{d t} \\
& =\frac{m}{2} \frac{d\left(v^{2}\right)}{d t}+\frac{m \omega^{2}}{2} \frac{d\left(x^{2}\right)}{d t} .
\end{aligned}
$$

We consider the case of a random dissipation function $f(H)$, due to fluctuations of the damping coefficient $b$ around its mean value. We replace $b$ by $b[1+\zeta(t)]$, where $\zeta(t)$ is noise with zero mean. We focus on the case where the noise is either Gaussian white noise or Poisson white noise (i.e., white shot noise). The dissipation function and the friction force $F$ now read

$$
\begin{aligned}
& f(H)=m b[1+\zeta(t)], \\
& F=-m b[1+\zeta(t)] v .
\end{aligned}
$$

The equations of motion for the fluctuating canonicaldissipative system are given by

$$
\begin{aligned}
& \frac{d x}{d t}=v, \\
& \frac{d v}{d t}=-b v-\omega^{2} x-b \zeta(t) v,
\end{aligned}
$$

and the energy dissipation rate is

$$
\frac{d E}{d t}=-m b[1+\zeta(t)] v^{2} .
$$

We combine Eqs. (2.13a) and (2.13b) to obtain the stochastic differential equation for the damped harmonic oscillator:

$$
\frac{d^{2} x}{d t^{2}}+b[1+\zeta(t)] \frac{d x}{d t}+\omega^{2} x=0 .
$$

Note that fluctuations in the damping coefficient lead to a stochastic differential equation with multiplicative noise.

Our main concern is how the fluctuations in the damping coefficient affect the stability of the oscillator. We employ three notions of stability. (i) Stability in the mean corresponds to $\langle x(t)\rangle \rightarrow 0$ as $t \rightarrow \infty$. (ii) Stability in the mean square corresponds to $\left\langle x(t)^{2}\right\rangle \rightarrow 0$ and $\left\langle v(t)^{2}\right\rangle \rightarrow 0$ as $t \rightarrow \infty$. Since the energy of the oscillator is given by $E=m v^{2} / 2+m \omega^{2} x^{2} / 2$, stability in the mean square is equivalent to energetic stability of the oscillator, i.e., $\langle E\rangle \rightarrow 0$ as $t \rightarrow \infty$. (iii) We define thermodynamic stability to correspond to a nonpositive mean rate of energy dissipation at all times, i.e., $\langle d E / d t\rangle \leqslant 0$ for $t \geqslant 0$. Averaging Eqs. (2.14) and (2.10), we find that

$$
\frac{d\langle E\rangle}{d t}=-m b\left\langle[1+\zeta(t)] v^{2}\right\rangle,
$$

or

$$
\frac{d\langle E\rangle}{d t}=\frac{m}{2} \frac{d}{d t}\left\langle v^{2}\right\rangle+\frac{m \omega^{2}}{2} \frac{d}{d t}\left\langle x^{2}\right\rangle .
$$




\section{DAMPED HARMONIC OSCILLATOR WITH GAUSSIAN WHITE NOISE}

In this section we consider the case that the damping coefficient $b$ undergoes uncorrelated Gaussian fluctuations. In other words, $\zeta(t)$ is a Gaussian white noise with

$$
\begin{gathered}
\langle\zeta(t)\rangle=0, \\
\left\langle\zeta(t) \zeta\left(t^{\prime}\right)\right\rangle=2 D \delta\left(t-t^{\prime}\right) .
\end{gathered}
$$

To determine the stability of the oscillator in the mean and the mean square, we need to calculate the mean values $\langle x\rangle$ and $\langle v\rangle$ and the mean-square values $\left\langle x^{2}\right\rangle$ and $\left\langle v^{2}\right\rangle$.

\section{A. Stability in the mean}

To obtain the mean values, we average Eqs. (2.13a) and (2.13b):

$$
\begin{aligned}
& \frac{d\langle x\rangle}{d t}=\langle v\rangle, \\
& \frac{d\langle v\rangle}{d t}=-b\langle v\rangle-\omega^{2}\langle x\rangle-b\langle\zeta v\rangle .
\end{aligned}
$$

This system of equations is not closed, and $\langle\zeta v\rangle$ must be determined separately. To this end we employ Novikov's theorem for Gaussian noise [22], which allows us to calculate the mean values of terms affected by the noise in a multiplicative way. The system (2.13) can be written in the general form

$$
\frac{d \boldsymbol{u}}{d t}=\boldsymbol{f}(\boldsymbol{u})+\boldsymbol{g}(\boldsymbol{u}) \zeta(t),
$$

where $\boldsymbol{u}=\left(u_{1}, \ldots, u_{n}\right)$. (All vectors should be read as column vectors. For ease of notation, we omit the transpose symbol for all vectors.) For Eq. (2.13) we identify

$$
\begin{gathered}
\boldsymbol{u}=\left(u_{1}, u_{2}\right)=(x, v), \\
\boldsymbol{f}(\boldsymbol{u})=\left(u_{2},-b u_{2}-\omega^{2} u_{1}\right), \\
\boldsymbol{g}(\boldsymbol{u})=\left(0,-b u_{2}\right) .
\end{gathered}
$$

Novikov's theorem for Eq. (3.4) states that

$$
\left\langle g_{i}(\boldsymbol{u}) \zeta(t)\right\rangle=D \sum_{j=1}^{n}\left\langle\frac{\partial g_{i}(\boldsymbol{u})}{\partial u_{j}} g_{j}(\boldsymbol{u})\right\rangle,
$$

where we have used Eq. (3.2) and the Stratonovich interpretation [23] for the stochastic calculus. Applying Eq. (3.8) to Eq. (3.7), we find

$$
\langle\zeta v\rangle=-D b\langle v\rangle
$$

which can be substituted into Eq. (3.3):

$$
\frac{d^{2}\langle x\rangle}{d t^{2}}+b(1-b D) \frac{d\langle x\rangle}{d t}+\omega^{2}\langle x\rangle=0 .
$$

Equation (3.10) is the equation of a damped harmonic oscillator for the mean position with an effective damping coefficient $b^{*}=b(1-b D)$ that depends on the noise intensity $D$. This result was also obtained by Gitterman $[14,18]$ by integrating formally Eq. (2.15). The mean position of the damped harmonic oscillator tends to 0 as $t \rightarrow \infty$ if $b D<1$. In other words, the stochastic oscillator is stable in the mean. However, stability in the mean is a weak notion of stability. Indeed, if $\langle x\rangle \rightarrow 0$ but the standard deviation grows as $t \rightarrow \infty$, then the oscillator can hardly be said to be stable in any practical sense. If $\left\langle x^{2}\right\rangle \rightarrow 0$ as $t \rightarrow \infty$, then the oscillator is stable in the mean square, which is a more relevant and restrictive concept in the dynamics of stochastic systems. In particular, if a system is stable in the mean square then it is stable in the mean. Further, if the oscillator is stable in the mean square, then it is energetically stable, as discussed above.

\section{B. Mean-square stability}

To determine $\left\langle x^{2}\right\rangle$ and $\left\langle v^{2}\right\rangle$, we multiply Eq. (2.13a) by $2 x$ and Eq. (2.13b) by $2 v$ :

$$
\begin{aligned}
& \frac{d x^{2}}{d t}=2 x \frac{d x}{d t}=2 x v, \\
& \frac{d v^{2}}{d t}=2 v \frac{d v}{d t}=-2 b v^{2}-2 b \zeta v^{2}-2 \omega^{2} x v .
\end{aligned}
$$

Multiplying Eqs. (2.13a) and (2.13b) by $v$ and $x$, respectively, and summing, we find

$$
\frac{d(x v)}{d t}=x \frac{d v}{d t}+v \frac{d x}{d t}=v^{2}-b x v-b \zeta x v-\omega^{2} x^{2} .
$$

Averaging Eqs. (3.11a), (3.11b), and (3.12), we obtain a hierarchy of ordinary differential equations for $\left\langle x^{2}\right\rangle,\left\langle v^{2}\right\rangle$, and $\langle x v\rangle$. To close the system, we need to determine $\left\langle\zeta v^{2}\right\rangle$ and $\langle\zeta x v\rangle$ and we employ again Novikov's theorem. Rewriting the systems (3.11) and (3.12) in the form of Eq. (3.4), where

$$
\begin{gathered}
\boldsymbol{u}=\left(u_{1}, u_{2}, u_{3}\right)=\left(x^{2}, v^{2}, x v\right), \\
f(\boldsymbol{u})=\left(2 u_{3},-2 b u_{2}-2 \omega^{2} u_{3},-b u_{3}-\omega^{2} u_{1}+u_{2}\right), \\
\boldsymbol{g}(\boldsymbol{u})=\left(0,-2 b u_{2},-b u_{3}\right),
\end{gathered}
$$

and applying Eq. (3.8) to Eq. (3.15), we find

$$
\begin{aligned}
& \left\langle\zeta v^{2}\right\rangle=-2 D b\left\langle v^{2}\right\rangle, \\
& \langle\zeta x v\rangle=-D b\langle x v\rangle .
\end{aligned}
$$

These expressions can be substituted into Eqs. (3.11b) and (3.12). The system of equations for the second moments can be written in the form

$$
\frac{d\langle\boldsymbol{u}\rangle}{d t}=\mathrm{M}\langle\boldsymbol{u}\rangle,
$$

where

$$
\mathbf{M}=\left(\begin{array}{ccc}
0 & 0 & 2 \\
0 & -2 b(1-2 b D) & -2 \omega^{2} \\
-\omega^{2} & 1 & -b(1-b D)
\end{array}\right) .
$$

The solution of Eq. (3.17) has the form

$$
\langle\boldsymbol{u}\rangle=\sum_{i=1}^{3} \boldsymbol{A}_{i} e^{\alpha_{i} t}
$$

The coefficients $\boldsymbol{A}_{i}$ must be determined from the initial conditions and depend on $b, D$, and $\omega$. The exponents $\alpha_{i}$ are the solutions of the characteristic polynomial $-\operatorname{det}(\mathrm{M}-\alpha \mathrm{l})=0$, where I is the $3 \times 3$ identity matrix:

$$
\alpha^{3}+c_{1} \alpha^{2}+c_{2} \alpha+c_{3}=0
$$


The coefficients are given by

$$
\begin{gathered}
c_{1}=b(3-5 D b), \\
c_{2}=4 \omega^{2}+2 b^{2}(1-2 b D)(1-b D), \\
c_{3}=4 \omega^{2} b(1-2 b D) .
\end{gathered}
$$

We are interested in the long time behavior of Eq. (3.19). We determine if the exponentials in Eq. (3.19) grow or decay with time by applying the Routh-Hurwitz theorem $[24,25]$ to the third-order polynomial (3.20). The Routh-Hurwitz conditions state that all the roots of the polynomial (3.20) have a negative real part if and only if $c_{3}>0, c_{1}>0$ and $c_{1} c_{2}-c_{3}>0$. This implies that if $b D<1 / 2$, then $\left\langle x^{2}\right\rangle \rightarrow 0$ and $\left\langle v^{2}\right\rangle \rightarrow 0$ as $t \rightarrow \infty$. Our result for the threshold of mean-square stability agrees with that for the oscillator studied in [18], where an uncorrelated additive Gaussian white noise force is present in addition to the random damping. We conclude that the additive noise does not affect the mean-square stability in this case.

If we consider the noise intensity $D$ as the control parameter, we arrive at the following conclusions. If $D$ is below $1 / 2 b$, the oscillator is stable both in the mean and in the mean square. If $D$ increases and falls between $1 / 2 b$ and $1 / b$, the oscillator is stable in the mean but unstable in the mean square. If $D$ further increases and exceeds $1 / b$, the oscillator is unstable both in the mean and in the mean square.

Let us now analyze the borderline cases $b D=1 / 2$ and $b D=1$ separately. As determined previously, if $b D=1 / 2$, then the oscillator is stable in the mean as confirmed by the equation for the mean position:

$$
\frac{d^{2}\langle x\rangle}{d t^{2}}+\frac{b}{2} \frac{d\langle x\rangle}{d t}+\omega^{2}\langle x\rangle=0 .
$$

The second moments $\left\langle x^{2}\right\rangle$ and $\left\langle v^{2}\right\rangle$ are obtained by solving the system (3.17) with the initial conditions $\left\langle x^{2}(0)\right\rangle=a^{2}$, $\left\langle v^{2}(0)\right\rangle=0$ and $\langle x v(0)\rangle=0$. If $b D=1 / 2$, then

$$
\begin{aligned}
& \left\langle x^{2}(t)\right\rangle=\frac{a^{2}}{2}\left\{1+e^{-b t / 4}\left[\cos (\tilde{\mu} t)+\frac{b}{4 \tilde{\mu}} \sin (\tilde{\mu} t)\right]\right\}, \\
& \left\langle v^{2}(t)\right\rangle=\frac{\omega^{2} a^{2}}{2}\left\{1-e^{-b t / 4}\left[\cos (\tilde{\mu} t)+\frac{b}{4 \tilde{\mu}} \sin (\tilde{\mu} t)\right]\right\},
\end{aligned}
$$

where

$$
\tilde{\mu}=\sqrt{4 \omega^{2}-\frac{b^{2}}{16}} .
$$

As $t \rightarrow \infty$, the second moments approach constant nonzero values, $\left\langle x^{2}(\infty)\right\rangle=a^{2} / 2$ and $\left\langle v^{2}(\infty)\right\rangle=\omega^{2} a^{2} / 2$. This implies that the oscillator is not stable in the mean square and $\langle E\rangle$ does not go to zero for $t \rightarrow \infty$.

If $b D=1$, the equation for the mean position is that of the undamped oscillator:

$$
\frac{d^{2}\langle x\rangle}{d t^{2}}+\omega^{2}\langle x\rangle=0 .
$$

The mean effect of the noise is to compensate the damping. The characteristic polynomial (3.20) in this case reads $\alpha^{3}-$ $2 b \alpha^{2}+4 \omega^{2} \alpha-4 \omega^{2} b=0$ and has a positive real solution. As $t \rightarrow \infty$, the second moments $\left\langle x^{2}\right\rangle$ and $\left\langle v^{2}\right\rangle$ behave as $\exp (\theta b t)$ where $\theta$ depends on $\omega / b$ and $1 \leqslant \theta \leqslant 2$. In particular, $\theta=2$ when $\omega \ll b$ and $\theta=1$ when $\omega \gg b$. In this case $\langle x\rangle$ oscillates between $-a$ and $a$ and the second moments diverge exponentially. The oscillator is unstable both in the mean and in the mean square or energetically.

\section{Thermodynamic stability}

As stated above, we say that the oscillator is thermodynamically stable if the average rate of energy dissipation is nonpositive at all times. For Gaussian white noise, Eqs. (2.14) and (3.16a) imply that

$$
\frac{d\langle E\rangle}{d t}=-m b(1-2 D b)\left\langle v^{2}\right\rangle .
$$

If $b D>1 / 2$, then $d\langle E\rangle / d t>0$ for $t \geqslant 0$ and the oscillator is thermodynamically unstable. In other words, the conditions for thermodynamic and mean-square instability coincide for Gaussian white noise. If the oscillator is stable in the mean square, then it dissipates energy; fluctuations in the damping coefficient do not change the nature of the dissipation function on average. If the oscillator is unstable in the mean square, then on average the noise pumps energy into the oscillator and renders it thermodynamically unstable.

\section{DAMPED HARMONIC OSCILLATOR WITH WHITE SHOT NOISE}

In this section we determine the stability conditions for the damped harmonic oscillator where the damping coefficient $b$ is subjected to white shot noise with zero mean. Gaussian white noise can take on negative values, which change the character of the friction term, namely, from dissipating energy to pumping energy. If the noise intensity is large enough, this effect leads to an instability of the oscillator. White shot noise has a finite lower bound, which allows us to control or even remove the effect of negative values of the fluctuating damping coefficient.

As for the case of Gaussian white noise, we first explore stability in the mean. White shot noise can be obtained as an appropriate limit of asymmetric dichotomous noise [26]. Let $\zeta(t)$ now be an asymmetric dichotomous noise that randomly switches between the values $-\Delta$ and $\Delta^{\prime}$. We assume that $\Delta^{\prime} \neq \Delta$, and both $\Delta$ and $\Delta^{\prime}$ are positive. We denote by $k_{\Delta}$ and $k_{\Delta^{\prime}}$ the transition probabilities per unit time between these two states. The average lifetime of each state is then given by $1 / k_{\Delta}$ and $1 / k_{\Delta^{\prime}}$, respectively. For the noise to have zero mean, we must impose $\Delta^{\prime} / k_{\Delta^{\prime}}=\Delta / k_{\Delta}$. The autocorrelation function of the asymmetric dichotomous noise is given by

$$
\left\langle\zeta(t) \zeta\left(t^{\prime}\right)\right\rangle=\sigma_{1} e^{-\lambda_{1}\left|t-t^{\prime}\right|},
$$

with $\sigma_{1}=\Delta \Delta^{\prime}$ and $\lambda_{1}=k_{\Delta}+k_{\Delta^{\prime}}$.

\section{A. Stability in the mean}

The starting point is again system (3.3). The term $\langle\zeta v\rangle$ now cannot be determined via Novikov's theorem because the noise is not Gaussian. However we can employ the 
Shapiro-Loginov formulas of differentiation for exponentially correlated random functions [27]:

$$
\begin{gathered}
\frac{d\langle\zeta v\rangle}{d t}=\left\langle\zeta \frac{d v}{d t}\right\rangle-\lambda_{1}\langle\zeta v\rangle, \\
\frac{d\langle\zeta x\rangle}{d t}=\langle\zeta v\rangle-\lambda_{1}\langle\zeta x\rangle .
\end{gathered}
$$

Combining Eqs. (4.2), (4.3), and (3.3), we find

$$
\begin{aligned}
\frac{d\langle x\rangle}{d t} & =\langle v\rangle, \\
\frac{d\langle v\rangle}{d t} & =-b\langle v\rangle-\omega^{2}\langle x\rangle-b\langle\zeta v\rangle, \\
\frac{d\langle\zeta v\rangle}{d t} & =-\left(b+\lambda_{1}\right)\langle\zeta v\rangle-b\left\langle\zeta^{2} v\right\rangle-\omega^{2}\langle\zeta x\rangle, \\
\frac{d\langle\zeta x\rangle}{d t} & =\langle\zeta v\rangle-\lambda_{1}\langle\zeta x\rangle .
\end{aligned}
$$

For dichotomous noise, $\left\langle\zeta^{2} v\right\rangle=\sigma_{1}\langle v\rangle+\lambda_{2}\langle\zeta v\rangle$, where $\lambda_{2}=\Delta^{\prime}-\Delta$, and the system (4.4) can be written as

$$
\begin{aligned}
\frac{d\langle x\rangle}{d t} & =\langle v\rangle, \\
\frac{d\langle v\rangle}{d t} & =-b\langle v\rangle-\omega^{2}\langle x\rangle-b\langle\zeta v\rangle, \\
\frac{d\langle\zeta v\rangle}{d t} & =-b \sigma_{1}\langle v\rangle-\left(b+\lambda_{1}+b \lambda_{2}\right)\langle\zeta v\rangle-\omega^{2}\langle\zeta x\rangle, \\
\frac{d\langle\zeta x\rangle}{d t} & =\langle\zeta v\rangle-\lambda_{1}\langle\zeta x\rangle .
\end{aligned}
$$

Asymmetric dichotomous noise converges to white shot noise of zero mean for $\Delta^{\prime} \rightarrow+\infty$ and $k_{\Delta^{\prime}} \rightarrow+\infty$ as the ratio $\Delta^{\prime} / k_{\Delta^{\prime}}$ remains constant [26]. We define $w_{0} \equiv \Delta^{\prime} / k_{\Delta^{\prime}}=$ $\Delta / k_{\Delta}$. We need to apply the white shot noise limit to the constants $\lambda_{1}, \lambda_{2}$, and $\sigma_{1}$. Since $\lambda_{1}=k_{\Delta}+k_{\Delta^{\prime}}, k_{\Delta^{\prime}} \rightarrow+\infty$ implies $\lambda_{1} \rightarrow+\infty$. On the other hand,

$$
\begin{gathered}
\frac{\sigma_{1}}{\lambda_{1}}=\frac{\Delta \Delta^{\prime}}{k_{\Delta}+k_{\Delta^{\prime}}}=\frac{\Delta^{\prime}}{k_{\Delta^{\prime}}} \frac{\Delta}{1+\frac{k_{\Delta}}{k_{\Delta^{\prime}}}} \\
=\stackrel{k_{\Delta^{\prime}}+\infty}{\longrightarrow} \frac{\Delta^{\prime}}{k_{\Delta^{\prime}}} \Delta=\frac{\Delta^{2}}{k_{\Delta}}, \\
\frac{\lambda_{2}}{\lambda_{1}}=\frac{\Delta^{\prime}-\Delta}{k_{\Delta}+k_{\Delta^{\prime}}} \stackrel{k_{\Delta^{\prime}} \rightarrow+\infty}{\Delta^{\prime} \rightarrow+\infty} \frac{\Delta^{\prime}}{k_{\Delta^{\prime}}}=\frac{\Delta}{k_{\Delta}} .
\end{gathered}
$$

This implies that $\sigma_{1} \rightarrow+\infty$ and $\lambda_{2} \rightarrow+\infty$ as $\lambda_{1} \rightarrow+\infty$.

White shot noise (Poisson white noise) is defined as $[12,28]$

$$
\zeta(t)=\sum_{i}^{n(t)} z_{i} \delta\left(t-t_{i}\right)-\beta\langle z\rangle,
$$

where $z_{i}$ are positive random amplitudes (weights) of the Dirac $\delta$ pulses (kicks) and the random instants $t_{i}$ are the arrival times of a Poissonian counting process $n(t)$ with parameter $\beta$. The time interval between two successive impulses is a random variable that is exponentially distributed according to $\beta e^{-\beta t}$. The parameter $\beta$ represents the mean number of impulses per unit time; i.e., $\beta^{-1}$ is the mean time between two successive impulses. The amplitudes are independent random variables that are also exponentially distributed according to $e^{-z / w_{0}} / w_{0}$, where $w_{0}$ is the mean amplitude. To establish the connection between the limit of the asymmetric dichotomous noise and the white shot noise, we need to express $\beta$ and $w_{0}$ in terms of $\Delta$ and $k_{\Delta}$. Since $\beta^{-1}$ is the mean time between two successive impulses and $k_{\Delta}^{-1}$ is the mean time in the state $\Delta$, both quantities must be the same, i.e.,

$$
\beta=k_{\Delta} .
$$

Equation (4.8) implies that the average and the correlation function of the white shot noise is given by $[12,28]$

$$
\begin{gathered}
\langle\zeta(t)\rangle=0, \\
\left\langle\zeta(t) \zeta\left(t^{\prime}\right)\right\rangle=2 \beta \omega_{0}^{2} \delta\left(t-t^{\prime}\right) .
\end{gathered}
$$

Taking the white shot noise limit, $\sigma_{1} \rightarrow+\infty$ and $\lambda_{1} \rightarrow+\infty$, of the correlation function (4.1) of the dichotomous noise, we find

$$
\begin{aligned}
\lim _{\sigma_{1}, \lambda_{1} \rightarrow+\infty}\left\langle\zeta(t) \zeta\left(t^{\prime}\right)\right\rangle & =\lim _{\sigma_{1}, \lambda_{1} \rightarrow+\infty} \sigma_{1} e^{-\lambda_{1}\left|t-t^{\prime}\right|} \\
& =\frac{2 \sigma_{1}}{\lambda_{1}} \delta\left(t-t^{\prime}\right) .
\end{aligned}
$$

Comparing Eqs. (4.12) and (4.11), we identify

$$
\frac{\sigma_{1}}{\lambda_{1}} \rightarrow \frac{\Delta^{2}}{k_{\Delta}}=\beta w_{0}^{2},
$$

and the noise intensity is given by $D=\beta w_{0}^{2}$. In contrast to zero-mean Gaussian white noise, zero-mean white shot noise is defined by two parameters, either $\beta$ and $w_{0}$, or the noise intensity $D$ and the non-Gaussian parameter $w_{0}$. In the limit $w_{0} \rightarrow 0$ with $D=\beta w_{0}^{2}=$ constant, white shot noise becomes Gaussian white noise with noise intensity $D$ [26]. Equations (4.9) and (4.13) establish the connection between the two noises. We conclude that in the white shot noise limit

$\sigma_{1} \rightarrow \beta w_{0}^{2} \lambda_{1} \quad$ and $\quad \lambda_{2} \rightarrow w_{0} \lambda_{1} \quad$ as $\quad \lambda_{1} \rightarrow+\infty$.

Applying the limit (4.14) to Eqs. (4.5c) and (4.5d), we find that $-b \beta w_{0}^{2}\langle v\rangle-\left(1+b w_{0}\right)\langle\zeta v\rangle=0$, i.e.,

$$
\langle\zeta v\rangle=-\frac{b \beta w_{0}^{2}}{1+b w_{0}}\langle v\rangle,
$$

and that

$$
\langle\zeta x\rangle=0 .
$$

We have performed numerical simulations of Eq. (2.15) with white shot noise to evaluate Eqs. (4.15) and (4.16). The results are shown in Fig. 1. The left- and right-hand sides of Eq. (4.15) have been determined independently from the simulations and have been plotted together to verify the validity of Eq. (4.15). The simulation results for $\langle\zeta x\rangle$ have been compared to $\langle x\rangle$ to verify Eq. (4.16). The figure demonstrates excellent agreement between the analytical and numerical results. Substituting Eqs. (4.15) and (4.16) into the system (4.5), we obtain

$$
\begin{gathered}
\frac{d\langle x\rangle}{d t}=\langle v\rangle, \\
\frac{d\langle v\rangle}{d t}=-b \frac{1+b w_{0}-b \beta w_{0}^{2}}{1+b w_{0}}\langle v\rangle-\omega^{2}\langle x\rangle .
\end{gathered}
$$



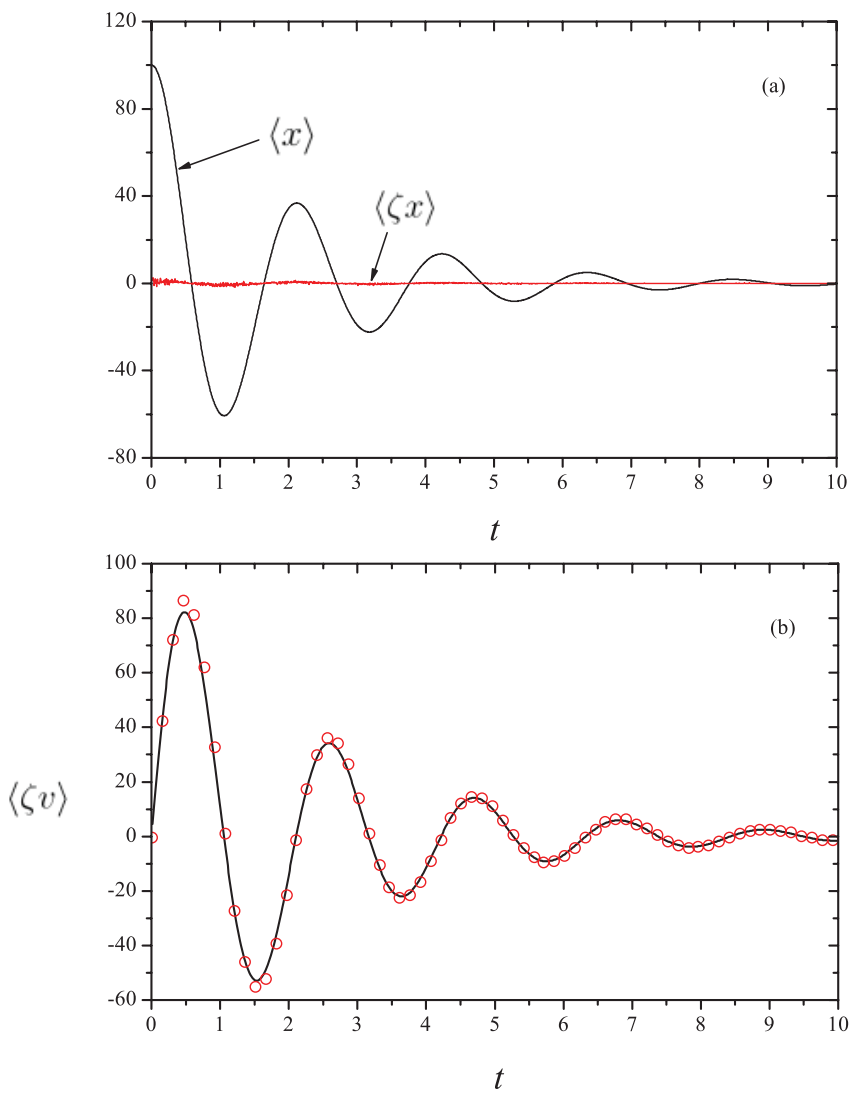

FIG. 1. (Color online) Results from numerical stochastic simulations to verify Eqs. (4.15) and (4.16). (a) $\langle x\rangle$ and $\langle\zeta x\rangle$ versus time. (b) Left-hand side (circles) and right-hand side (solid line) of Eq. (4.15). We have performed averages over $10^{6}$ realizations, $\delta t=0.005, w_{0}=0.2, \omega=3, b=1$, and $\beta=5$.

These equations can be rewritten as an equation for the mean position of the damped oscillator equation with an effective damping coefficient:

$$
\frac{d^{2}\langle x\rangle}{d t^{2}}+b^{*} \frac{d\langle x\rangle}{d t}+\omega^{2}\langle x\rangle=0
$$

where

$$
b^{*}=b\left(1-\frac{b \beta w_{0}^{2}}{1+b w_{0}}\right)
$$

Note that in the Gaussian white noise limit $w_{0} \rightarrow 0$ with $\beta w_{0}^{2} \rightarrow D$, we recover Eq. (3.10) as expected. The sign of $b^{*}$ defines the condition

$$
\mathrm{C}_{0} \equiv \beta b w_{0}^{2}-b w_{0}-1 .
$$

If $\mathrm{C}_{0}<0$, the oscillator subjected to white shot noise is stable in the mean, $\langle x\rangle \rightarrow 0$ as $t \rightarrow \infty$. The lower bound for the fluctuations of the damping coefficient $b[1+\zeta(t)]$ is given by $b(1-\Delta)$. Equations (4.9) and (4.13) imply that $\Delta=\beta w_{0}$, and the condition (4.21) turns into

$$
0<\Delta<\Delta^{*}=\frac{1}{2}\left(1+\sqrt{1+\frac{4 \beta}{b}}\right) .
$$

If $\Delta<1$, the white shot noise does not change the nature of the friction term. The fluctuating damping coefficient is never negative and the fluctuating rate of energy dissipation is never positive. In contrast to Gaussian white noise, there are no random instances where the friction term represents an energy pump. As expected, the oscillator is stable in the mean if subjected to white shot noise with a nonnegative lower bound. Indeed, since $\Delta^{*}>1$, the condition $\Delta<1$ ensures that $\langle x\rangle \rightarrow 0$ as $t \rightarrow \infty$. On the other hand, condition (4.22) also clearly implies that negative fluctuations do not compromise the stability of the oscillator in the mean, if their amplitude is sufficiently small. The mean position of the oscillator still goes to zero, $\langle x\rangle \rightarrow 0$ as $t \rightarrow \infty$, for $1<\Delta<\Delta^{*}$. However, as discussed in Sec. III, stability in the mean is a weak notion of stability, and we analyze the second moments to determine the conditions for which the oscillator is mean-square stable.

\section{B. Mean-square stability}

Using the Shapiro-Loginov formula for the averages $\left\langle\zeta v^{2}\right\rangle$, $\langle\zeta x v\rangle$, and $\left\langle\zeta x^{2}\right\rangle$

$$
\begin{gathered}
\frac{d\left\langle\zeta v^{2}\right\rangle}{d t}=\left\langle\zeta \frac{d v^{2}}{d t}\right\rangle-\lambda_{1}\left\langle\zeta v^{2}\right\rangle, \\
\frac{d\left\langle\zeta x^{2}\right\rangle}{d t}=\left\langle\zeta \frac{d x^{2}}{d t}\right\rangle-\lambda_{1}\left\langle\zeta x^{2}\right\rangle, \\
\frac{d\langle\zeta x v\rangle}{d t}=\left\langle\zeta \frac{d(x v)}{d t}\right\rangle-\lambda_{1}\langle\zeta x v\rangle,
\end{gathered}
$$

Eqs. (3.11a), (3.11b), (3.12), and

$$
\begin{gathered}
\left\langle\zeta^{2} x v\right\rangle=\sigma_{1}\langle x v\rangle+\lambda_{2}\langle\zeta x v\rangle, \\
\left\langle\zeta^{2} v^{2}\right\rangle=\sigma_{1}\left\langle v^{2}\right\rangle+\lambda_{2}\left\langle\zeta v^{2}\right\rangle
\end{gathered}
$$

we write the system of six ordinary differential equations for $\left\langle x^{2}\right\rangle,\left\langle v^{2}\right\rangle,\langle x v\rangle,\left\langle\zeta v^{2}\right\rangle,\langle\zeta x v\rangle$, and $\left\langle\zeta x^{2}\right\rangle$ in the form

$$
\begin{aligned}
\frac{d\left\langle x^{2}\right\rangle}{d t}= & 2\langle x v\rangle, \\
\frac{d\left\langle v^{2}\right\rangle}{d t}= & -2 b\left\langle v^{2}\right\rangle-2 b\left\langle\zeta v^{2}\right\rangle-2 \omega^{2}\langle x v\rangle, \\
\frac{d\langle x v\rangle}{d t}= & \left\langle v^{2}\right\rangle-b\langle x v\rangle-b\langle x v \zeta\rangle-\omega^{2}\langle x\rangle, \\
\frac{d\left\langle\zeta v^{2}\right\rangle}{d t}= & -2 b \sigma_{1}\left\langle v^{2}\right\rangle-\left[2 b\left(1+\lambda_{2}\right)+\lambda_{1}\right]\left\langle\zeta v^{2}\right\rangle \\
\frac{d\langle\zeta x v\rangle}{d t}= & -b \sigma_{1}\langle x v\rangle-\left[b\left(1+\lambda_{2}\right)+\lambda_{1}\right]\langle\zeta x v\rangle \\
& +\left\langle\zeta v^{2}\right\rangle-\omega^{2}\left\langle\zeta x^{2}\right\rangle, \\
\frac{d\left\langle\zeta x^{2}\right\rangle}{d t}= & 2\langle\zeta x v\rangle-\lambda_{1}\left\langle\zeta x^{2}\right\rangle .
\end{aligned}
$$$$
\frac{d\left\langle\zeta v^{2}\right\rangle}{d t}=-2 b \sigma_{1}\left\langle v^{2}\right\rangle-\left[2 b\left(1+\lambda_{2}\right)+\lambda_{1}\right]\left\langle\zeta v^{2}\right\rangle-2 \omega^{2}\langle x\rangle,
$$

Applying the limit (4.14) to Eq. (4.28d), we find that $-2 b \beta w_{0}^{2}\left\langle v^{2}\right\rangle-\left(2 b w_{0}+1\right)\left\langle\zeta v^{2}\right\rangle=0$, i.e.,

$$
\left\langle\zeta v^{2}\right\rangle=-\frac{2 b \beta w_{0}^{2}}{2 b w_{0}+1}\left\langle v^{2}\right\rangle
$$

Applying the limit (4.14) to Eq. (4.28e), we find that $-b \beta w_{0}^{2}\langle x v\rangle-\left(b w_{0}+1\right)\langle\zeta x v\rangle=0$, i.e.,

$$
\langle\zeta x v\rangle=-\frac{b \beta w_{0}^{2}}{b w_{0}+1}\langle x v\rangle .
$$



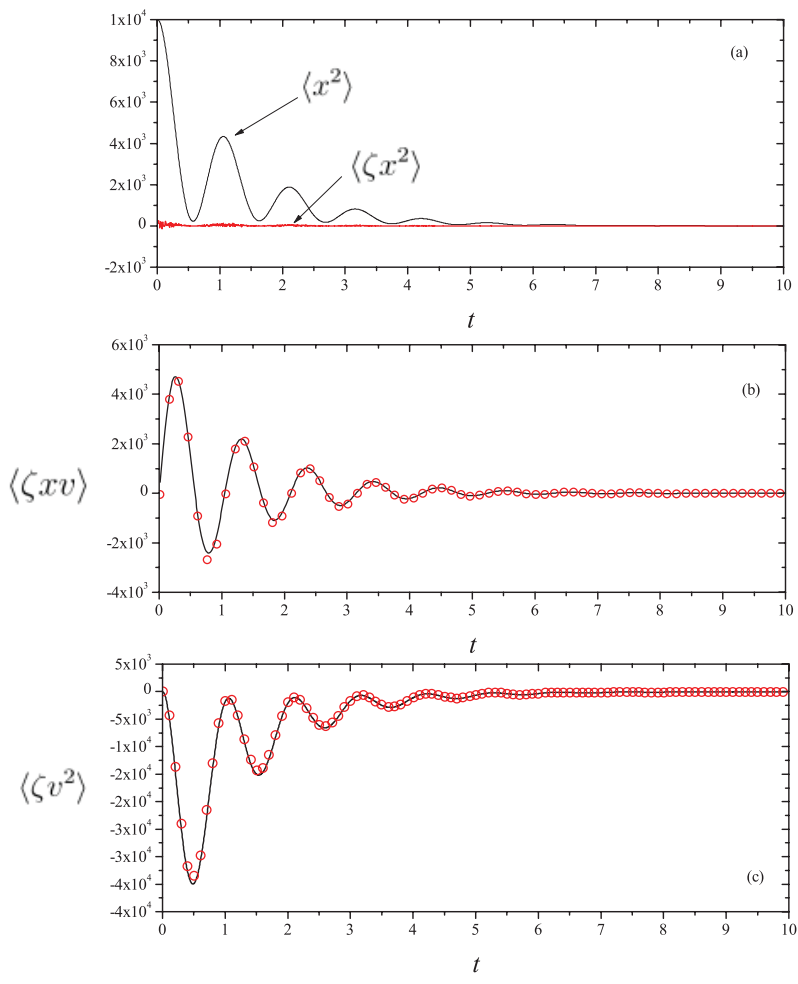

FIG. 2. (Color online) Results from numerical stochastic simulations to verify Eqs. (4.29), (4.30), and (4.31). (a) $\left\langle x^{2}\right\rangle$ and $\left\langle\zeta x^{2}\right\rangle$ versus time. (b) Left-hand side (circles) and right-hand side (solid line) of Eq. (4.30). (c) Left-hand side (circles) and right-hand side (solid line) of Eq. (4.29). We have performed averages over $10^{6}$ realizations, $\delta t=0.005, w_{0}=0.2, \omega=3, b=1$, and $\beta=5$.

Application of the limit (4.14) to Eq. (4.28f) yields

$$
\left\langle\zeta x^{2}\right\rangle=0
$$

In Fig. 2 we plot the left- and right-hand sides of Eqs. (4.29) and (4.30) determined via numerical simulations. To verify the validity of Eq. (4.31), we plot $\left\langle\zeta x^{2}\right\rangle$ together with $\left\langle x^{2}\right\rangle$. The figure demonstrates again excellent agreement between the numerical and analytical results. Substituting Eqs. (4.29), (4.30), and (4.31) into the system (4.28) we obtain

$$
\begin{aligned}
& \frac{d\left\langle x^{2}\right\rangle}{d t}=2\langle x v\rangle, \\
& \frac{d\left\langle v^{2}\right\rangle}{d t}=-2 b \frac{2 b w_{0}+1-2 b \beta w_{0}^{2}}{2 b w_{0}+1}\left\langle v^{2}\right\rangle-2 \omega^{2}\langle x v\rangle, \\
& \frac{d\langle x v\rangle}{d t}=\left\langle v^{2}\right\rangle-b \frac{b w_{0}+1-b \beta w_{0}^{2}}{b w_{0}+1}\langle x v\rangle-\omega^{2}\langle x\rangle .
\end{aligned}
$$

The solution of the system (4.32) can be written in the form of Eq. (3.19) with

$$
\begin{aligned}
\alpha_{1}= & -2 b \frac{1+2 b w_{0}-2 b \beta w_{0}^{2}}{1+2 b w_{0}}, \\
\alpha_{2,3}= & -\frac{b}{2} \frac{b w_{0}+1-b \beta w_{0}^{2}}{b w_{0}+1} \\
& \pm \frac{1}{2} \sqrt{b^{2}\left(\frac{b w_{0}+1-b \beta w_{0}^{2}}{b w_{0}+1}\right)^{2}-8 \omega^{2} .}
\end{aligned}
$$

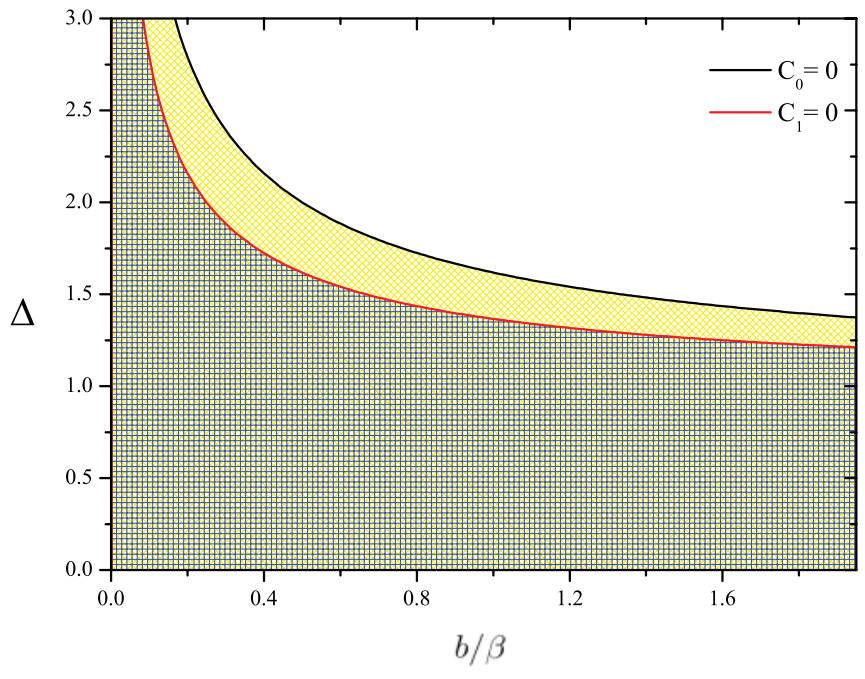

FIG. 3. (Color online) Stability diagram in the $\Delta-b / \beta$ plane for the damped oscillator under white shot noise for $\omega / \beta=0.1$. We represent the critical curves provided by the conditions $\mathrm{C}_{0}=0$ and $\mathrm{C}_{1}=0$. In the upper (white) region, the oscillator is unstable in the mean and in the mean square, $\langle x\rangle \rightarrow \infty$ and $\left\langle x^{2}\right\rangle \rightarrow \infty$ as $t \rightarrow \infty$. In the intermediate (yellow) region, it is stable in the mean but unstable in the mean square, $\langle x\rangle \rightarrow 0$ and $\left\langle x^{2}\right\rangle \rightarrow \infty$ as $t \rightarrow \infty$. In the lower (blue) region, it is stable both in the mean and in the mean square, $\langle x\rangle \rightarrow 0$ and $\left\langle x^{2}\right\rangle \rightarrow 0$ as $t \rightarrow \infty$.

Note that the sign of $\alpha_{1}$ depends on the sign of the new condition

$$
\mathrm{C}_{1} \equiv 2 b \beta w_{0}^{2}-2 b w_{0}-1
$$

while the sign of $\alpha_{2}$ and $\alpha_{3}$ depends on the sign of $\mathrm{C}_{0}$. In Fig. 3 we have plotted conditions $C_{0}$ and $C_{1}$. In the blue region, below the curve $\mathrm{C}_{1}=0$, we have $\alpha_{1,2,3}<0$ and the oscillator is stable in both the mean and the mean square. In the region lying between $\mathrm{C}_{0}=0$ and $\mathrm{C}_{1}=0$, the oscillator is unstable in the mean square due to $\alpha_{1}>0$. In the region above $\mathrm{C}_{0}=0$, we have $\alpha_{1,2,3}>0$ and the oscillator is unstable in the mean as well as in the mean square. Note that in the Gaussian limit $w_{0} \rightarrow 0$ with $D=\beta w_{0}^{2}=$ constant, condition (4.35) reduces to $b D<1 / 2$, and the condition for stability in the mean, Eq. (4.21), turns into $b D<1$, in agreement with our previous results.

\section{Thermodynamic stability}

If $\Delta=\beta w_{0}<1$, then the friction force $F$ is a true friction force at all instances; the oscillator always dissipates energy. This can also be clearly seen in Fig. 3, where the region below $\Delta=1$ corresponds to stability for any value of $b$ and $\beta$. If $\Delta=\beta w_{0}>1$, or if $\zeta(t)$ is Gaussian white noise, the fluctuations change the character of $F$ at random instances. If the fluctuations lead to negative values of $b(t)=b[1+\zeta(t)]$, the fluctuating energy dissipation rate becomes positive. In other words, the damping term corresponds to an energy pump. We showed in Sec. III C that stability in the mean square and thermodynamic stability coincide for Gaussian white noise. This turns out to be true also for white shot noise. Indeed we 
find for the case of white shot noise from Eqs. (2.14) and (4.29) that

$$
\frac{d\langle E\rangle}{d t}=-m b \frac{1+2 b w_{0}-2 b \beta w_{0}^{2}}{1+2 b w_{0}}\left\langle v^{2}\right\rangle .
$$

The sign of $d\langle E\rangle / d t$ for $t \geqslant 0$ depends only on condition $\mathrm{C}_{1}$, which is the condition for the stability in the mean square.

\section{CONCLUSIONS}

We have studied the instabilities of the stochastic linear damped oscillator induced by a fluctuating damping parameter. Due to the multiplicative character of the noise, the oscillator is unstable in the mean and in the mean square if the noise intensity is high enough. Given the form of the Hamiltonian, stability in the mean square is more physically relevant than stability in the mean, since the former guarantees the energetic stability of the damped oscillator. We have considered a second physically relevant stability criterion, namely, thermodynamic stability. If the oscillator is indeed damped due to friction forces for mechanical systems, or due to other dissipative mechanisms in other types of applications that can be modelled by simple harmonic motion, then the average rate of energy dissipation should be nonpositive. This is a priori a stronger stability criterion than mean-square stability. The latter is an asymptotic criterion; the second moments must vanish as $t \rightarrow \infty$. Thermodynamic stability requires that the average energy dissipation rate be nonpositive at all times. Further, this rate depends not only on $\left\langle v^{2}\right\rangle$ but also on the cross correlation between the velocity and the noise, $\left\langle\zeta v^{2}\right\rangle$.

We have derived analytically conditions for stability in the mean, in the mean square, and for thermodynamic stability for two types of white noise, namely, for Gaussian white noise and white shot noise. If the damping coefficient is subjected to Gaussian white noise, then the fluctuations inevitably change the nature of the friction force. Gaussian white noise has no lower bound, and negative fluctuations, which occur with the same probability as positive fluctuations, turn the friction mechanism into an energy pump. On the other hand, white shot noise does have a lower bound and provides us with a way to assess the effect of negative values of the fluctuating damping coefficient on the stability of the oscillator.

If the noise intensity is considered as a control parameter, then the dynamics of the stochastic oscillator undergoes two bifurcations between three possible phases: (i) stable (stable both in the mean and in the mean square), (ii) thermodynamically unstable (stable in the mean but unstable in the mean square), and (iii) unstable (unstable both in the mean and in the mean square). For low values of the noise intensity, the oscillator is stable. When the noise intensity increases and reaches the lowest stability threshold, the oscillator becomes thermodynamically unstable. When the noise intensity reaches the second threshold, the oscillator becomes unstable.

We have shown analytically that stability in the mean square, which is equivalent to energetic stability, coincides with thermodynamic stability for Gaussian white noise and Poisson white noise. This is an unexpected result, because thermodynamic stability does not depend solely on the meansquare values of the position and the velocity. As mentioned above, it is also affected by the cross correlation between the velocity and the noise. This poses the interesting open problem of delineating the family of noises for which energetic and thermodynamic stability coincide.

\section{ACKNOWLEDGMENTS}

This research has been partially supported by the Generalitat de Catalunya with Grant No. SGR 2009-164 (VM, DC) and by Ministerio de Ciencia e Innovación with Grant No. FIS2009-13370-C02-01 (VM).
[1] M. Gitterman, The Noisy Oscillator: The First Hundred Years, From Einstein Until Now (World Scientific, Singapore, 2005).

[2] G. E. Uhlenbeck and L. S. Ornstein, Phys. Rev. 36, 823 (1930).

[3] M. C. Wang and G. E. Uhlenbeck, Rev. Mod. Phys. 17, 323 (1945).

[4] G. Papanicolau and J. B. Keller, SIAM J. Appl. Math. 21, 287 (1971).

[5] R. C. Bourret, U. Frisch, and A. Pouquet, Physica 65, 303 (1973).

[6] K. Lindenberg, V. Seshadri, and B. J. West, Phys. Rev. A 22, 2171 (1980).

[7] K. Mallick and P. Marcq, J. Phys. A 37, 4769 (2004).

[8] M. Gitterman, Phys. Rev. E 67, 057103 (2003).

[9] M. Gitterman, Physica A 352, 309 (2005).

[10] M. Gitterman and V. I. Klyatskin, Phys. Rev. E 81, 051139 (2010).

[11] M. Gitterman, J. Phys.: Conf. Ser. 248, 012049 (2010).

[12] J. Łuczka, P. Talkner, and P. Hänggi, Physica A 278, 18 (2000).

[13] P. Talkner and J. Łuczka, in Stochastic Processes in Physics, Chemistry, and Biology, edited by J. Freund and T. Pöschel, Vol. 557 of Lecture Notes in Physics (Springer, Berlin, 2000), pp. 85-96.

[14] M. Gitterman, Phys. Rev. E 69, 041101 (2004).
[15] D. Jasnow and E. Gerjuoy, Phys. Rev. A 11, 340 (1975).

[16] B. J. West and V. Seshadri, J. Geophys. Res. 86, 4293 (1981).

[17] J. Grue and B. Øksendal, Stoch. Proc. Appl. 68, 113 (1997).

[18] M. Gitterman, J. Phys. A 37, 5729 (2004).

[19] H. Haken, Z. Phys. A 263, 267 (1973).

[20] W. Ebeling, in Stochastic Processes in Physics, Chemistry, and Biology, edited by J. A. Freund and T. Pöschel, Vol. 557 of Lecture Notes in Physics (Springer, Berlin, 2000), pp. 390-399.

[21] F. Schweitzer, W. Ebeling, and B. Tilch, Phys. Rev. E 64, 021110 (2001).

[22] E. A. Novikov, Zh. Eksp. Teor. Fiz. 47, 1919 (1964) [Sov. Phys. JETP 20, 1290 (1965)].

[23] W. Horsthemke and R. Lefever, Noise-Induced Transitions. Theory and Applications in Physics, Chemistry, and Biology (Springer-Verlag, Berlin, 1984).

[24] F. R. Gantmacher, The Theory of Matrices, Vol. 2 (AMS Chelsea, Providence, 1959).

[25] J. D. Murray, Mathematical Biology I: An Introduction (Springer, New York, 2002).

[26] C. Van den Broeck, J. Stat. Phys. 31, 467 (1983).

[27] V. E. Shapiro and V. M. Loginov, Physica A 91, 563 (1978).

[28] P. Hänggi, Z. Phys. B 36, 271 (1980). 